\title{
La phénoménologie à l'épreuve des sciences humaines
}

B. Frère, S. Laoureux dir. 


\section{Introduction}

L'ouvrage que l'on va lire ${ }^{1}$ trouve son impulsion initiale dans un constat: l'usage répété qui est fait d'une démarche ou d'une méthode phénoménologique par toute une série de chercheurs opérant dans différentes disciplines des sciences humaines. Inspirés des travaux de l'école philosophique de phénoménologie, les travaux de ces chercheurs témoignent des potentialités que représentent ses ressources pour ces disciplines. Cet usage se fait le plus souvent dans un souci opératoire plutôt qu'avec l'ambition de répondre à des questions de fondation, qui sur le terrain sont peu pertinentes pour les scientifiques et pour la pratique concrète de leur discipline. Depuis plusieurs années, ce souci répond un peu partout dans les sciences humaines au désir de rompre avec des approches surplombantes du monde, des espaces, des sujets. En deçà des grandes structures qui font la vie (de l'inconscient, du social, du politique) l'on cherche à saisir ce qui fait sens ici et maintenant, avant de postuler l'existence de structures générales qu'il s'agirait de mettre au jour. Le singulier est gros de l'universel mais pour le montrer il convient de plonger dans les vécus humains qui se tissent de rapports immédiats au corps, aux animaux, aux choses et aux autres. La phénoménologie semble être la perspective la plus pertinente pour décortiquer dans toute leur finesse la matière de ces vécus et leur teneur généralisable.

Plusieurs de ses dimensions sont conjointement abordées dans cet ouvrage. Il s'agit d'abord d'évaluer la spécificité des usages de la phénoménologie dans les sciences humaines dès lors qu'elles se trouvent confrontées à une problématique qui exige d'elles une prise sur le monde vécu. Ainsi, les praticiens présents dans cet ouvrage (sociologie, anthropologie, psychiatrie, psychanalyse, géographie, théorie sociale) détaillent en quel sens ils revendiquent un point de vue et une approche proprement phénoménologiques face à des difficultés que les

\footnotetext{
1 Les textes recueillis constituent les actes d'un colloque tenu à l'Université de Liège les 9 et 10 mai 2007 sous le titre «Usages de méthodes phénoménologiques en sciences humaines ». Il était organisé par l'Institut des sciences humaines et sociales, le Groupe Pragma - sociologie théorique et politique -, l'unité de recherche "Phénoménologies" du Département de Philosophie (Université de Liège) en collaboration avec le Département de Philosophie de l'Université de Namur.
} 
méthodes usuelles de leurs disciplines respectives ne parviennent pas à surmonter. Dans cette perspective, ils exposent plus systématiquement ce qui fait l'attrait spécifique de la phénoménologie pour leurs disciplines et ce que peut signifier pour eux de «faire de la phénoménologie » en usant de concepts tels que la conscience intentionnelle, la présence au monde, le corps propre, l'épochè ou encore plus simplement le «Je». Pour ce faire, ces contributions s'efforcent d'illustrer leurs propos en recourant à des exemples concrets, des « terrains » ou des « cas » bien spécifiques. Elles indiquent également quels sont leurs univers théoriques de références et les auteurs phénoménologues qui sont privilégiés dans leurs perspectives. En cela d'ailleurs l'intérêt majeur de chacune d'entre elle prise même individuellement est de montrer combien des philosophes et des philosophies que les non initiés ont l'habitude de juger ésotériques peuvent en fait constituer de redoutables outils de compréhension du monde actuel. En somme, grâce à la richesse des matériaux et théories propres à leurs disciplines respectives et au savant dosage de phénoménologie qu'ils leur insufflent, les contributeurs de cet ouvrage cherchent à relever modestement rien moins que l'un des plus grands défis de la philosophie dès lors qu'elle sort de sa tour d'ivoire pour se frotter aux contingences de la réalité. 
Nathalie Depraz, dans un premier chapitre, ouvre les hostilités en cherchant à saisir l'utilité du regard phénoménologique - et de l'acte élémentaire que constitue pour lui la réduction (épochè) -pour un ensemble de disciplines que la philosophie qualifie de «positives »: la théologie, la psychiatrie et l'anthropologie. Pour ce faire, elle procède à un détour préalable. En effet, pour en faire une méthode utilisable empiriquement, Nathalie Depraz reconnaît avoir dû modifier la méthode phénoménologique ou, plus précisément, avoir dû redéfinir les trois dimensions fondamentales de l'épochè pour mettre en évidence leur vertu opératoire : la conversion, la suspension et la variation. Ainsi redéfinie, l'épochè devient un geste de recherche empirique qui permet de se mettre en lien direct avec l'attitude vécue des sujets. Dans la pratique de la prière du coeur, observée chez certains moines orthodoxes de Grèce et de Russie, l'épochè devient la pratique incarnée de relation à soi comme ouverture en soi de l'autre infini. Dans le cas de la psychiatrie, elle vient également requalifier une attitude qui est déjà celle des médecins (ouverture, disposition, accueil, attention à la personne, implication dans le vécu), leur donnant par la même l'occasion de nommer enfin une pratique déjà opérante et, dans le même temps, la possibilité de la revisiter et de la retravailler. Enfin, dans le cas de l'observation ethnographique de la communauté greque d'Istanbul, l'épochè permet de décrire le mode d'être ensemble sans emprise ni appétit de connaissance possessive de l'autre (d'où émerge une intelligibilité du vécu partagé), mode d'être qui est le propre de cette situation connue de bien des ethnologues dans laquelle « observateur » et « observés » s'observent en réalité mutuellement.

La contribution d'Emmanuelle Tulle, quant à elle, s'installe sur le terrain sociologique du vieillissement. Selon elle les méthodes sociologiques traditionnelles dans ce champ (comme celle de Leder) et la biogérontologie (d'inspiration cartésienne) ne sont jamais venues à bout d'un paradoxe que l'auteure suggère aujourd'hui de confronter à sa relecture de la phénoménologie de Merleau-Ponty. En effet, lors d'une enquête sur la façon dont les sportifs se vivent comme catégorie sociale, elle a pu remarquer que les plus vieillissants d'entre eux 'prétendent' en réalité souvent ne pas se sentir vieillir et ce alors même que toutes leurs performances physiques leur prouvent le contraire. Ces énoncés sont sous-tendus par une appréhension de la relation corps-âme conçue comme un idéal type d'harmonie parfaite. Le vieillissement met en danger cet équilibre et provoque, d'une certaine manière, une crise ontologique. Il apparaît que, pour y parer, la seule solution est d'exclure le corps de l'expérience réflexive et de donner priorité à un «moi » pur, désincarné. Voilà qui laisse le sens du soi face au vieillissement dans une position insoutenable puisque le corps, conçu par ailleurs comme le socle empirique de son ontologie, se retrouve abandonné, au fur et à mesure 
que nous vieillissons, pour protéger une identité que l'on voudrait être sans âge. Or les coureurs ne procèdent pas à ce rejet, loin s'en faut. Comment dès lors expliquer leur persévérance et leur plaisir dans leur sport alors que le monde social (Leder) en général et le monde médical en particulier (biogérontologie) les stigmatisent comme «déclinants », «non performants », bref comme « vieux »?

La contribution de Jean-Marie Gauthier, pédopsychiatre, docteur en psychologie clinique, s'installe résolument dans une posture critique à l'égard du paradigme de la psychanalyse classique en s'appuyant sur des ressources phénoménologiques. C'est en repartant de sa pratique en psychiatrie de l'enfant qu'il indique comment celle-ci interroge certaines des conceptions les mieux ancrées de la psychanalyse classique. Et c'est à partir d'une référence à la phénoménologie (en particulier celle de Sartre, mais aussi de MerleauPonty ou de Ricœur) qu'il est possible d'apporter, selon lui, des réponses à ces interrogations fondamentales laissées en suspens par le paradigme classique. Son texte propose de la sorte un heureux et très éclairant va-et-vient entre références à son travail empirique de praticien en psychopathologie de l'enfant, critique qu'il est possible d'adresser à partir de là à la psychanalyse adulte et ressources théoriques offertes par certains auteurs phénoménologues. Jean-Marie Gauthier envisage d'abord la place du couple corps/espace, fondamentale pour la psychiatrie de l'enfant alors que le psychanalyste classique l'ignore. Sa contribution s'intéresse également de près à la question de l'inconscient en insistant tout particulièrement sur le nouveau regard qu'il est nécessaire de jeter sur son pendant, souvent peu étudié pour lui-même : la conscience. Il conviendrait, selon lui, de faire place à une conception plus « dialectique » qui pourrait notamment expliquer ce que signifie exactement la «prise de conscience » essentielle dans le processus thérapeutique, et pourtant laissée dans un vague théorique. Enfin, sa contribution montre comment c'est par une référence à la phénoménologie sartrienne, et plus particulièrement à la place que celle-ci ménage au récit de vie et au parcours biographique, que l'on peut apporter des éléments de réponse aux questions laissées en suspens par la psychanalyse quant au corps, à l'espace ou à la conscience. Une telle référence permet notamment de ne plus ignorer les racines biologiques et sociohistoriques des individus.

Dans sa contribution, le géographe André-Frédéric Hoyaux insiste sur deux dimensions qui lui semblent pertinentes dans la référence à la phénoménologie. Elle lui permet d'abord d'entretenir un rapport critique à sa propre discipline - et à tout le moins à 
plusieurs de ses «écoles ». En ce sens, la première partie de son analyse se présente comme un parcours historique dans sa discipline qui lui permet d'en présenter les grandes tendances épistémologiques : de celle qui n'accorde aucune réalité fondatrice à l'espace, le confinant dans un rôle d'« espace-support», jusqu'à celle qui s'inscrit dans une perspective phénoménologique faisant du monde environnant un cadre de vie potentiel autant naturel que social dont dispose l'individu, en passant par celle qui s'inscrit dans la mouvance poststructuraliste ou encore celle qui hérite des comportementalistes. Ce bref parcours lui permet ensuite de spécifier en quoi l'approche phénoménologie féconde sa discipline. Sur le plan méthodologique, tout d'abord, en concevant de façon spécifique la relation de l'homme à l'espace et qui débouche in fine sur l'élaboration d'une réflexion globale sur le concept d'habiter. Sur le plan épistémologique, ensuite, en renouvelant, essentiellement à partir de la phénoménologie herméneutique, la question du rapport du chercheur à son «objet»: l'habitant. Mettant en évidence ces deux tournants géographiques (épistémologique et méthodologique) impulsé par la référence à la phénoménologie, André-Frédéric Hoyaux termine alors sa contribution en suivant de façon plus ponctuelle, deux aspects sur lesquels la référence phénoménologique (abordée essentiellement à partir de l'œuvre heideggérienne dans ce cadre) en géographie présente de belles potentialités : la question de l'éloignement et la question de l'authenticité.

Frédéric Keck, adoptant le regard de l'anthropologue, s'interroge sur le besoin contemporain de représenter publiquement les conditions de vie des animaux autrement qu'à travers les grilles de la zootechnie. En effet, depuis la crise de la vache folle et celle de la dioxine nos sociétés semblent s'interroger à nouveaux frais sur la façon dont nous avons longtemps considéré l'animal comme un objet, une simple machine à produire. Ces crises et les menaces qu'elles ont fait peser sur l'humain nous rappellent violemment qu'en deçà du rapport objectif entretenu avec les animaux, nous partageons avec eux un monde commun. Mais cette participation commune, dans le schème de rationalité instrumentale qui est le nôtre aujourd'hui, s'avère bien difficile à représenter. C'est pourtant le pari que la phénoménologie peut nous aider à relever. Pour le démontrer, Frédéric Keck s'appuie sur un usage croisé des travaux de Levy-Bruhl et de Husserl. Selon le premier en effet les hommes sont susceptibles de s'identifier aux animaux présents dans leur environnement. Les identités « homme» et « animal » ne se pensent pas par contradiction (ce qui induit une altérité laissant au premier le droit de disposer du second) mais par «participation ». En conférant à la notion le sens de «Wesenschau » (vision des essences) que lui attribuait la phénoménologie de Husserl et qui 
renvoie à la possibilité de l'insertion de l'intelligible dans le sensible (la «metexis» platonicienne) il devient possible d'émettre une critique constructive du " monde technique », conçu comme représentation homogénéisante des êtres de l'environnement à travers les grilles de la rationalité instrumentale des humains. Mais cette critique ne va pas de soi. En effet, selon Frédéric Keck les premières tentatives de la sociologie, notamment celles de Porcher ou de Chateauraynaud, tout excellentes qu'elles soient, échouent partiellement à rendre compte de cette participation. En effet, le point de vue reste tantôt celui de l'éleveur (Porcher) tantôt celui de l'expert vétérinaire (Chateauraynaud). Dès lors y a-t-il une relation affective aux animaux qui soit capable de faire raisonner une strate d'intentionnalité commune plus profonde susceptible de nous aider à comprendre la malaise humain à l'égard de leur traitement?

Patrick Baert travaille à offrir un nouveau programme de recherche à la théorie sociale afin de nous aider à rénover notre façon de faire des sciences sociales : le néo-pragmatisme. Pour ce faire, deux sources théoriques lui semblent fondamentales : le pragmatisme américain et la phénoménologie continentale. En France, le pragmatisme américain connaît un certain retour en grâce depuis une quinzaine d'année, notamment grâce à l'école sociologique du même nom ${ }^{2}$. Pour solidifier ses qualités opératoires, Patrick Beart entend assurer son commerce avec la phénoménologie. Plus précisément avec trois de ses penseurs. Sartre, d'abord, peut permettre au (néo-) pragmatisme de penser une notion qui lui est chère, celle de liberté. Le pour-soi sartrien renvoie en effet les individus à la liberté qu'ils ont de se définir eux-mêmes et de renier les catégories exclusives sous lesquelles ils sont perçus (l'être en soi). La phénoménologie de l'altérité lévinasienne ensuite est convoquée pour asseoir ce programme pragmatique. Tout en tenant la position husserlienne selon laquelle l'intentionnalité représente le monde à travers la médiation de la conscience, il insiste sur le fait que la rencontre avec le monde extérieur est faite d'un échange dynamique dans lequel le sujet s'avère engagé avec autrui et affecté par lui. Or précisément, le projet pragmatiste veut pour sa part concevoir les rencontres de différentes formes de vie comme autant d'opportunités pour nous de nous redécrire, de nous réévaluer et de nous réinventer nousmêmes. Dans le même sens, la notion gadamerienne de compréhension authentique, au coeur

2 . L'école de sociologie pragmatique trouve son origine dans la seconde moitié des années 80 dans les travaux de Luc Boltanski et Laurent Thévenot. Alors appelée sociologie de la critique, elle s'est ensuite progressivement qualifiée de sociologie pragmatique. A partir de la fin des années 1990 les auteurs ayant emboité le pas à ce courant ont progressivement tracé les ponts nécessaires avec la pragmatique américaines, donnant lieu à divers publications théoriques (citer). 
du tournant phénoménologique qu'il fait subir à l'herméneutique, vient conclure cette tentative de fonder phénoménologiquement le projet pragmatique. S'accordant à Husserl sur l'idée que les sciences naturelles ne peuvent émerger que dans et marquée par le "monde de la vie », Gadamer refuse que les critères scientifiques d'objectivité soient les standards de la connaissance en général et accepte que d'autres normes et critères puissent servir la compréhension authentique. Enfin, à titre d'exemple, Baert évoque les travaux de sociologie citoyenne de Burawoy pour indiquer ce qui pourrait être une esquisse de recherche néopragmatique renforcée par ces apports théoriques.

Enfin, Bruno Frère et Sébastien Laoureux, tentent, dans le chapitre qui ferme l'ouvrage, de s'interroger sur les potentialités mais aussi les limites sociologiques de la phénoménologie. Ils repartent de la pensée du fondateur de la perspective phénoménologique en sociologie - Alfred Schütz. Tout en s'efforçant d'indiquer certaines des impasses théoriques auxquelles mène le projet schützéen d'appliquer le transcendantal à l'empirique (lié tout particulièrement au maintien d'une perspective égologique), ils retiennent les potentialités de la posture épistémologique qui est la sienne. C'est une telle posture que l'on retrouve en effet à l'œuvre aujourd'hui dans la sociologie dite pragmatique, et qui a déjà donné nombre de travaux particulièrement stimulants. Ensuite, au-delà de Schütz, et en se référant prioritairement à la pensée sociologique d'inspiration phénoménologique de Max Scheler, ils indiquent comment il est possible de se réapproprier dans une perspective sociologique des concepts et outils phénoménologiques qui dépassent la perspective « subjectiviste » de Husserl et qui serait encore celle de Schütz. Sans plus se poser la question du «transcendantal» ce sont les notions d'intentionnalité (Husserl) et d'états d'esprits (Scheler) qui retiennent leur attention. En effet, selon eux, la sociologie gagnerait beaucoup à décrypter les visées intentionnelles qui sont celles des acteurs dans l'action et ce afin de relever que les oppositions et les clivages dans la vie sociale peuvent en partie être compris comme le résultat de représentations du monde divergentes et de constitutions subjectives de sens objectifs opposés.

Comme on le voit, l'ambition des textes rassemblés ici n'est pas de présenter une étude exhaustive sur la question, mais bien d'en opérer un ensemble de clarifications théoriques et surtout de relever les avancées considérables que permet la phénoménologie dès lors qu'on accepte d'en faire réellement une méthode susceptible d'être mobilisée sur les terrains empiriques de disciplines aussi variées que la géographie ou la psychiatrie. D’autres 
disciplines auraient pu être convoquées, d'autres débats évoqués. Nous espérons, néanmoins, qu'un des acquis indéniables de cet ouvrage sera d'avoir proposé une première illustration très concrète de possibles approches phénoménologiques en sciences humaines A l'heure où les diverses formes de structuralisme se sont essoufflées, où le constructivisme a montré ses propres limites et où le renouveau semble venir de paradigmes qui sont très proches d'elle à divers égards (comme le pragmatisme), il ne fait pas de doute que la phénoménologie doit être réinvestie. Il s'agit en somme d'en faire ce qui n'avait pas encore été proposé explicitement: un outil d'analyse du monde et des choses, ici et maintenant. Cet outil recèle bien des promesses d'innovation, toutes disciplines confondues. La philosophie a toujours été à cet égard une source vivante pour les sciences humaines en général. Gageons qu'elle reviendra à son tour de plus bel à la phénoménologie après avoir fréquenté ses mises à l'épreuve pratiques pour approfondir encore ses concepts et son appréhension dynamique du monde.

Bruno Frère et Sébastien Laoureux 